\title{
Inhaltsverzeichnis
}

\section{Global Data Synchronization - Lösungsansatz für das überbetriebliche Produktstammdatenmanagement zwischen Konsumgüterindustrie und Handel? ................................. 3}

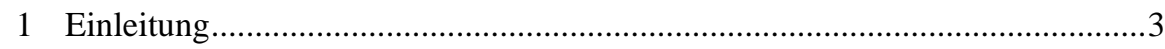

2 Kooperation zwischen Industrie und Handel ....................................................

3 Produktstammdatenmanagement als Basis elektronischer Kooperation.............4

4 Lösungsansätze für das elektronische Stammdatenmanagement zwischen

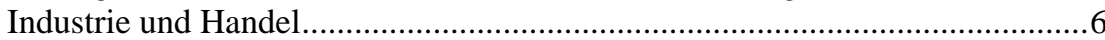

4.1 Austauschformen: bilateral vs. multilateral ...........................................6

4.2 Das Konzept der globalen Stammdatensynchronisation .............................

5 Überbetriebliches Produktstammdatenmanagement in der Praxis: Eine

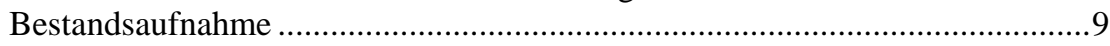

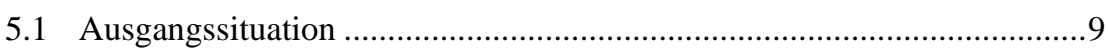

5.2 Überbetriebliche Stammdatenprozesse: mehrstufige Interaktion ...............9

5.3 Systemarchitektur: Datenaustausch per Spreadsheet................................11

6 Global Data Synchronization: Bewertung des Lösungsansatzes .......................13

6.1 Standardisierungsgrad und Anschlussfähigkeit als

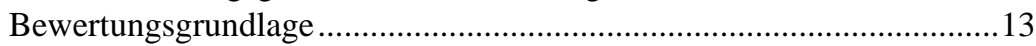

6.2 Gestaltungsebene: Daten und Funktionen .............................................13

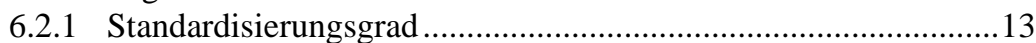

6.2.2 Anschlussfähigkeit .......................................................................14

6.3 Gestaltungsebene: Prozesse im Stammdatenmanagement ........................16

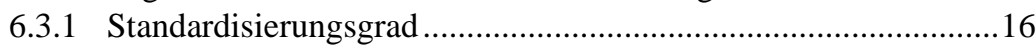

6.3.2 Anschlussfähigkeit ................................................................16

6.4 Gestaltungsebene: Geschäftsstandards ..................................................17

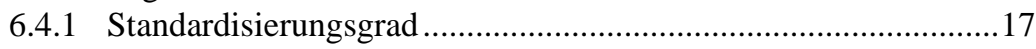

6.4.2 Anschlussfähigkeit ................................................................... 
II Inhaltsverzeichnis

7 Zusammenfassung und Ausblick ......................................................................18

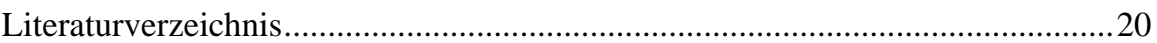

Autorenverzeichnis.............................................................24 


\section{Global Data Synchronization - Lösungsansatz für das überbetriebliche Produktstammdatenmanagement zwischen Konsumgüterindustrie und Handel?}

Jan W. Schemm, Christine Legner, Hubert Österle

\section{Einleitung}

Abgestimmte Produktstammdaten bilden die Grundlage elektronischer Geschäftsbeziehungen zwischen Handels- und Industrieunternehmen. Obwohl Handel und Industrie sich schon vergleichsweise lange mit dem elektronischen Austausch von Produktdaten beschäftigen, konnten sich weder der EDI-basierte, bilaterale Stammdatenaustausch noch der multilaterale Ansatz über Datenpools durchsetzen. Seit Mitte 2004 verfolgt das Global Data Synchronization Network (GDSN) einen neuen Lösungsansatz, mit dem Ziel, einen m:n-fähigen elektronischen Produktstammdatenaustausch zu etablieren. Die Ankündigungen von führenden Handelsunternehmen wie WAL-MART, AHOLD oder TESCO (Loderhose 2005a, 2005b, Rode 2005c) sowie Konsumgüterherstellern wie NestLÉ, Procter \& GAMBLE oder KRAFT Foods (Rode 2005a, 2005b) schüren ebenso wie die aktuelle Konsolidierung unter den Stammdatenpools (Garf u. Romanow 2005) die Erwartungen. Der vorliegende Beitrag untersucht die Fragestellung, inwieweit GDSN die notwendigen Grundlagen für ein m:n-fähiges Produktstammdatenmanagement zwischen Industrie und Handel liefert. Die Autoren führen zunächst in die Fragestellung des überbetrieblichen Stammdatenmanagements ein und erläutern das GDSN-Konzept. Anhand des überbetrieblichen Stammdatenaustausches zwischen zwei Herstellern und einem Handelsunternehmen illustriert der Beitrag die IstSituation sowie die Anforderungen an einen m:n-fähigen elektronischen Stammdatenaustausch. Basierend auf den Ergebnissen dieser Fallstudie bewerten die Autoren GDSN einerseits in Bezug auf den Reifegrad der Standardisierung, und andererseits in Bezug auf die Anschlussfähigkeit von Industrie- und Handelsunternehmen an die sich entwickelnden Standards. 


\section{Kooperation zwischen Industrie und Handel}

Eine aktuelle Studie unter 33 europäischen Einzelhändlern von (Thonemann et al. 2005) identifiziert Supply Chain Champions, welche die zentrale Herausforderung im Handel vergleichsweise besser als ihre Verfolger meistern: die führenden Handelsunternehmen erreichen höheren Kundenservice (gemessen anhand der Regalverfügbarkeit) bei niedrigerem Logistikaufwand (gemessen anhand der Logistikkosten und der Reichweite des Gesamtbestands). Eine Strategie zur Renditesteigerung liegt für Händler in der intensiveren elektronischen Zusammenarbeit mit ihren Zulieferern aus der Konsumgüterindustrie. Entsprechende Kooperationsmodelle zwischen Industrie und Handel werden seit den 90er Jahren unter dem Begriff Efficient Consumer Response (ECR) subsumiert.

\begin{tabular}{|l|l|l|l|}
\hline $\begin{array}{c}\text { Kooperations- } \\
\text { bereich } \\
\text { (ECR-Begriff) }\end{array}$ & $\begin{array}{c}\text { Kooperations- } \\
\text { prozess }\end{array}$ & Kooperationsziel & $\begin{array}{c}\text { Kooperations- } \\
\text { aktivitäten }\end{array}$ \\
\hline $\begin{array}{l}\text { Supply Chain } \\
\text { Management } \\
\text { (Supply Side) }\end{array}$ & $\begin{array}{c}\text { - Beschaffung- } \\
\text { Distribution }\end{array}$ & $\begin{array}{l}\text { Kostenreduzie- } \\
\text { rung }\end{array}$ & $\begin{array}{l}\bullet \text { Beschaffungs- } \\
\text { logistik } \\
\bullet \text { Transportlogistik }\end{array}$ \\
\hline $\begin{array}{l}\text { Category Mana- } \\
\text { gemen } \\
\text { (Demand Side) }\end{array}$ & $\begin{array}{l}\text { Distribution- } \\
\text { Absatz }\end{array}$ & $\begin{array}{l}\text { Umsatzsteige- } \\
\text { rung }\end{array}$ & $\begin{array}{l}\text { - Produktpolitik } \\
\text { Preis- und } \\
\text { Aktionspolitik } \\
\text { Platzierungs- } \\
\text { politik }\end{array}$ \\
\hline
\end{tabular}

Tab. 1: Kooperationen zwischen Industrie- und Handelsunternehmen (Becker u. Schütte 2004, Holzkämper 1999)

Tab. 1 zeigt die beiden Kooperationsbereiche: während im Supply Chain Management (kooperative Logistik) vor allem die Kostenreduzierung durch Verbesserungen in der Beschaffungs- und Distributionslogistik im Vordergrund steht, werden im Category Management (kooperatives Marketing) Umsatzsteigerungen durch gezielte Produkt-, Platzierungs-, Preis- und Aktionspolitik erzielt.

\section{Produktstammdatenmanagement als Basis elektronischer Kooperation}

Elektronische Kooperationsprozesse, wie sie ECR vorschlägt, erfordern unternehmensübergreifend abgestimmte Produktstammdaten (Alt u. Fleisch 1999). Dies begründet die Anstrengungen auf Seiten von Industrie und Handel, einen elektronischen Stammdatenaustausch zu etablieren. Der betriebswirtschaftliche Nutzen entsteht dabei in zwei Dimensionen. Erstens lassen sich durch Beseitigung von Medienbrüchen und Automatisierung die hohen Aufwände in den Stammdatenprozessen reduzieren (Global Commerce Initiative u. Capgemini 2005, Grocery 
Manufacturers of America et al. 2003). Zweitens reduzieren schnell verfügbare und fehlerfreie Produktstammdaten Fehler und damit Kosten in den Nutzungsprozessen, z. B. in der Logistik (Global Commerce Initiative u. Capgemini 2005, Grocery Manufacturers of America et al. 2003). Sie beschleunigen indirekt auch die Produkteinführung und erhöhen die Regalverfügbarkeit, was letztlich zu Umsatzsteigerungen führt (Corsten u. Gruen 2003, Global Commerce Initiative u. Capgemini 2005, Grocery Manufacturers of America et al. 2003).

Stammdaten beschreiben die Kernentitäten eines Unternehmens (White et al. 2006). Sie sind elementarer Bestandteil von betrieblichen Informationssystemen und umfassen Attribute, die im Gegensatz zu Bewegungsdaten

- nur in Ausnahmefällen oder zumindest selten Änderungen unterliegen (Mertens 1995, Wedekind 1997),

- von Bewegungsdaten häufig zur Beschreibung von Geschäftsvorfällen referenziert werden (White et al. 2006) und

- in unterschiedliche Sichten unterteilt sind, die funktionale bzw. organisatorische Gruppierungen der Attribute darstellen (Leukel 2004, Loos 1999, Schütte u. Vering 2004).

Produkt- bzw. Artikelstammdaten stellen neben Kunden-, Lieferanten-, Betriebsmittel-, Finanz- und Personalstammdaten die wichtigsten Stammdaten einer integrierten Informationsverarbeitung dar (Mertens 1995, White et al. 2004). Produktstammdatenmanagement umfasst alle Aufgaben zur Planung, Steuerung und Kontrolle der betrieblichen und technischen Organisation von Produktstammdaten in Unternehmen. Die Kernleistung dieses Unterstützungsprozesses (Hess 1996) besteht in der Versorgung der geschäftlichen Leistungsprozesse mit den benötigten Stammdaten. Produktstammdatenmanagement lässt sich durch die folgenden typischen Prozessmerkmale charakterisieren (Österle 1995):

- Produktstammdatenmanagement unterteilt sich in die Mikroprozesse Produktanlage, Produktänderung, Produktausphasierung.

- Die Aufgaben im Produktstammdatenmanagement sind typischerweise über mehrere organisatorische Einheiten wie bspw. Einkauf, Produktion, Warenwirtschaft und Vertrieb verteilt.

- IT-Anwendungen wie bspw. Systeme für Enterprise Resource Planning (ERP) oder Product Lifecycle Management (PLM) unterstützen traditionell die Aufgaben im Produktstammdatenmanagement. Neuerdings setzen Unternehmen auch auf dedizierte Systeme für Master Data Management (MDM) bzw. Product Information Management (PIM) (Lucas-Nülle 2005, White 2005).

- Das Produktstammdatenmanagement wird über Führungsgrößen wie bspw. die Anzahl unvollständiger Stammdatensätze oder die Durchlaufzeit eines Anlageprozesses im Rahmen einer Prozessführung gesteuert. 


\section{Lösungsansätze für das elektronische Stammdatenmanagement zwischen Industrie und Handel}

\subsection{Austauschformen: bilateral vs. multilateral}

Heute bieten sich für Industrie- und Handelsunternehmen prinzipiell zwei Varianten für den Austausch von Produktstammdaten an (vgl. Abb. 1): bilateral unter Nutzung von Standards oder multilateral über einen Stammdatenpool (Hertel et al. 2005).

Die EDI-basierten, standardisierten Nachrichtentypen PRODAT (Produktstammdaten) und PRICAT (Preisliste / Katalog) des EDIFACT-Subsets EANCOM bieten eine seit längerem etablierte Option für den Produktstammdatenabgleich (Hertel et al. 2005). Eine neuer Alternative bilden die XML-Standards der Standardisierungsorganisation GS1 (GS1 2006c). Diese Nachrichtentypen stellen plattformunabhängige, XML-basierte Weiterentwicklungen der EANCOM-Standards dar. Trotz der Nutzung der genannten Standards laufen direkte Austauschformen in den meisten Fällen aufgrund der Interpretationsspielräume bzw. des Anpassungsbedarfs auf 1:1 oder 1:n-Verbindungen hinaus (Senger 2004).

Bilateraler Stammdatenaustausch

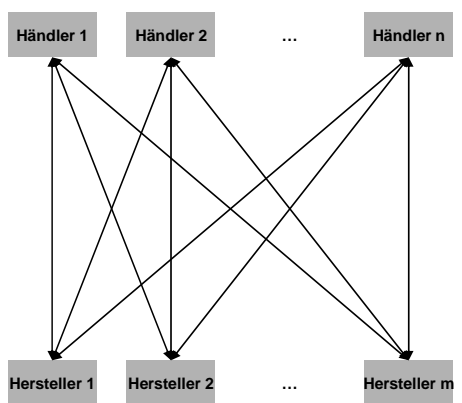

Austausch über Stammdatenpool

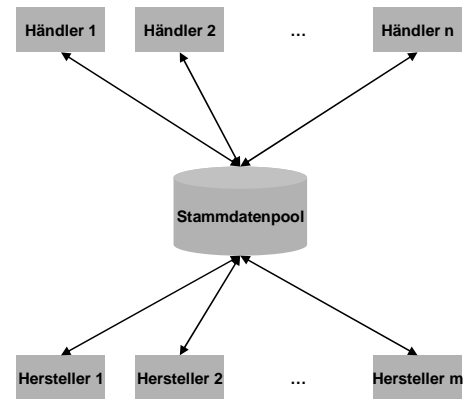

Abb. 1: Formen des überbetrieblichen elektronischen Stammdatenaustauschs

Im multilateralen Fall tauschen die Geschäftspartner die Stammdatennachrichten nicht direkt, sondern über einen Stammdatenpool aus. Stammdatenpools sind Exchanges (Corsten u. Hofstetter 2001, Sparks u. Wagner 2003), die als Intermediäre stammdatenbezogene Prozesse, Funktionen und Daten standardisieren. Durch diese de facto Standardisierung reduzieren Stammdatenpools die Schnittstellenanzahl und vereinfachen m:n-Verbindungen in Geschäftsnetzwerken vgl. (Kagermann u. Österle 2006). Beispiele für Stammdatenpools sind das deutsche Unternehmen SINFOS sowie die US-amerikanischen Player Agentrics (Fusion von WWRE und GNX) und 1Sync (Fusion von Transora und UCCnet) (Garf u. 
Romanow 2005). Stammdatenpools bieten ihren Kunden i.d.R. mehrere parallele Anschlussvarianten (bspw. EDI-basiert über PRICAT, XML-basiert oder manuell über eine Web-Schnittstelle) an und realisieren im Idealfall einen m:n-fähigen Stammdatenaustausch mit nur einer Schnittstelle pro angeschlossenem Unternehmen. Die angestrebte m:n-Fähigkeit ist insofern zu hinterfragen, als heute weltweit ca. 25 Datenpools operieren (GS1 2006e). Das Konzept der globalen Datensynchronisation soll dieses Problem lösen.

\subsection{Das Konzept der globalen Stammdatensynchronisation}

Globale Stammdatensynchronisation hat das Ziel des globalen Austauschs von Produktinformationen über Unternehmensgrenzen hinweg in nahezu Echtzeit (Bowling et al. 2004, Nakatani et al. 2006). Das Global Data Synchronization Network (GDSN) soll als Umsetzungskonzept das Problem der mangelnden m:nFähigkeit im mulitlateralen Stammdatenaustausch lösen. GDSN ist ein von den Standardisierungsorganisationen GS1, UCC und GCI entwickeltes Konzept zur Föderation von Stammdatenpools über zusätzliche Standards und ein globales Verzeichnis (GS1 2004). Abb. 2 stellt den Nachrichten- und Datenfluss beim Prozess des überbetrieblichen Produktstammdatenaustauschs im GDSN dar.

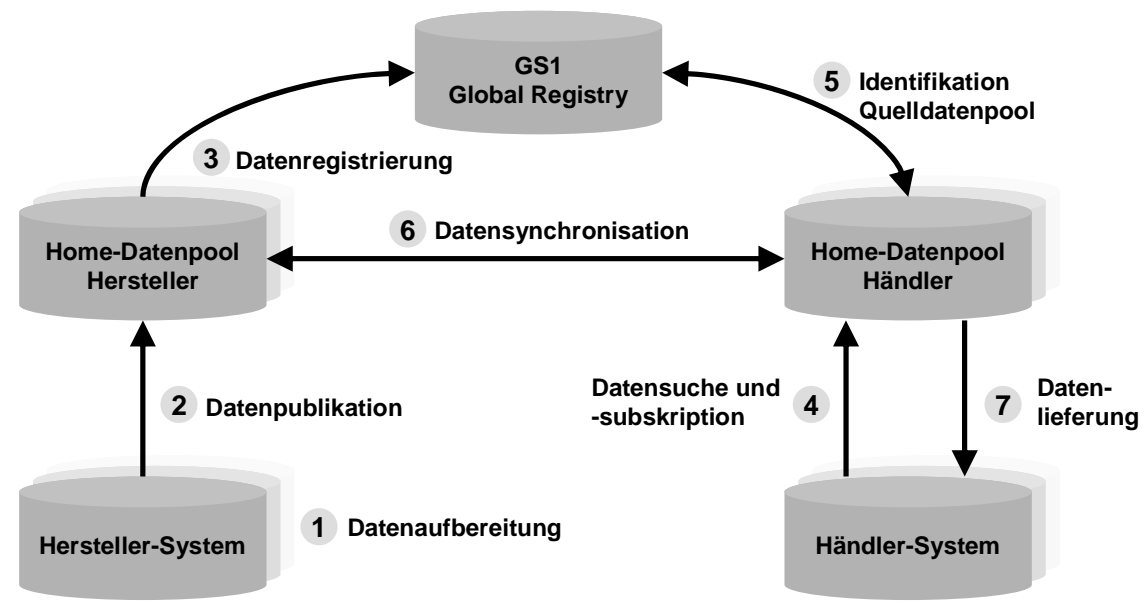

Abb. 2: Ablauf des Stammdatenaustauschs im GDSN

Der illustrierte Stammdatenaustausch lässt sich in 7 Phasen unterteilen (GS1 2004):

1. Datenaufbereitung: Der Hersteller beschreibt seine Produktstammdaten unter Nutzung der GDSN-Standards (vgl. Tab. 2).

2. Datenpublikation: Der Hersteller übermittelt die Produktdaten an einen GDSN-zertifizierten Home-Datenpool. 
3. Datenregistrierung: Der Datenpool des Herstellers übermittelt eine Teilmenge der Produktdaten (insbesondere GLN, GTIN, GPC, Zielmarkt und die Poolkennung) an das von GS1 betriebene globale Verzeichnis.

4. Datensuche und -subskription: Das Handelsunternehmen sucht über seinen Home-Datenpool nach Produktstammdaten und abonniert diese Daten über eine Kombination aus GLN, GTIN, GPC und Zielmarkt.

5. Identifikation Quelldatenpool: Der Home-Datenpool des Händlers ermittelt über das zentrale Verzeichnis den Datenpool, der die Produktstammdaten des gewünschten Herstellers hält.

6. Datensynchronisation: Der Datenpool des Herstellers übermittelt die gewünschten Daten an den Datenpool des Händlers.

7. Datenlieferung: Der Händler erhält die abonnierten Produktstammdaten über seinen Home-Datenpool.

\begin{tabular}{|l|l|l|}
\hline \multicolumn{1}{|c|}{ Kategorie } & \multicolumn{1}{|c|}{ Name } & \multicolumn{1}{c|}{ Beschreibung } \\
\hline $\begin{array}{l}\text { Partner- } \\
\text { identifikation }\end{array}$ & $\begin{array}{l}\text { Global Location } \\
\text { Number (GLN) }\end{array}$ & $\begin{array}{l}\text { Die GLN identifiziert die volle Unternehmens- } \\
\text { bezeichnung inkl. Adresse und wird im } \\
\text { deutschsprachigen Raum auch als Internatio- } \\
\text { nale Lokationsnummer (ILN) bezeichnet. }\end{array}$ \\
\hline $\begin{array}{l}\text { Produkt- } \\
\text { identifikation }\end{array}$ & $\begin{array}{l}\text { Global Trade } \\
\text { Item Identifica- } \\
\text { tion (GTIN) }\end{array}$ & $\begin{array}{l}\text { Die GTIN ist ein neuer Sammelbegriff für die } \\
\text { Internationale Artikelnummer (EAN) und identi- } \\
\text { fiziert Produkte und logistische Einheiten welt- } \\
\text { weit eindeutig. }\end{array}$ \\
\hline $\begin{array}{l}\text { Produkt- } \\
\text { klassifikation }\end{array}$ & $\begin{array}{l}\text { Global Product } \\
\text { Classification } \\
\text { (GPC) }\end{array}$ & $\begin{array}{l}\text { Die GPC definiert eine standardisierte Sorti- } \\
\text { mentshierarchie zur Klassifikation von Produk- } \\
\text { ten. }\end{array}$ \\
\hline $\begin{array}{l}\text { Produkt- } \\
\text { beschreibung }\end{array}$ & $\begin{array}{l}\text { Data Model for } \\
\text { Trade Item }\end{array}$ & $\begin{array}{l}\text { GS1 definiert in einem zentralen Lexikon bzw. } \\
\text { Verzeichnis für Daten- und Nachrichtenstan- } \\
\text { dards, dem Global Data Dictionary (GDD), u. a. } \\
\text { ein Produktdatenmodell. Enthalten sind eine } \\
\text { Menge von Kernattributen sowie ergänzende } \\
\text { Attribute (bspw. marktspezifisch, sortiments- } \\
\text { spezifisch, beziehungsspezifisch) }\end{array}$ \\
\hline
\end{tabular}

Tab. 2: Standards im GDSN

Über den beschriebenen Prozess werden neben neuen Produktdaten über das Publish-Subscribe-Prinzip auch Änderungen und Ausphasierungen kommuniziert, wodurch das GDSN die elementaren Mikroprozesse des überbetrieblichen Produktstammdatenmanagements abdeckt. Das von GS1 betriebene globale Verzeichnis ist seit August 2004 operativ und GDSN umfasst aktuell ca. 25 zertifi- 
zierte Datenpools (GS1 2006e). Erste Statistiken ${ }^{1}$ lassen eine langsame Annahme des Konzepts durch den Markt vermuten, eine Abschätzung des Umfangs der operativen Nutzung im Stammdatenmanagement ist jedoch anhand der vorliegenden Zahlen nicht möglich.

\section{5 Überbetriebliches Produktstammdatenmanagement in der Praxis: Eine Bestandsaufnahme}

\subsection{Ausgangssituation}

Die folgende Analyse beschreibt die Ist-Situation im überbetrieblichen Produktstammdatenmanagement zwischen zwei europäischen Konsumgüterherstellern und einem Handelsunternehmen. Sie betrachtet insbesondere die Stammdatenprozesse sowie deren Systemunterstützung und leitet daraus Anforderungen an ein überbetriebliches Stammdatenmanagement ab. Obwohl die Analyse sich auf den konkreten Fall der beteiligten Unternehmen bezieht, bilden die Ergebnisse nach Einschätzung der Autoren ein typisches und generalisierbares Bild der aktuellen Situation des überbetrieblichen Produktstammdatenmanagements in europäischen Hersteller-Händler-Beziehungen. Nachfolgende Gespräche mit weiteren Vertretern aus Industrie- und Handelsunternehmen lieferten erste Bestätigungen der abgeleiteten Kernaussagen.

\section{2 Überbetriebliche Stammdatenprozesse: mehrstufige Interaktion}

Prozessablauf, Art und Umfang der ausgetauschten Stammdaten sowie die ITUnterstützung der Stammdatenprozesse zwischen Industrie und Handel variieren in Abhängigkeit von Produktsortiment (bspw. Lebensmittel, Bekleidung, Möbel) und Produkttyp (Marken- vs. Eigenprodukt). Dennoch lässt sich aus den typischen stammdatenbezogenen Aufgaben auf Seiten des Handels und der Hersteller ein überbetrieblicher Produktstammdatenprozess ableiten. Dieser ist in Abb. 3 für die Produktneuanlage eines über Vendor Managed Inventory (VMI) bewirtschafteten Markenprodukts im Sortiment Beauty/Personal Care/Hygiene dargestellt:

- Der Prozess beinhaltet auf Industrie- und Handelsseite interne Aufgabenfolgen, in die jeweils mehrere unterschiedliche Organisationseinheiten involviert

1 Im Februar 2006 waren ca. 3'800 GLNs und ca. 480'000 GTINs im globalen Verzeichnis registriert (GS1 2006b). 
sind. Auf Herstellerseite sind ein zentrales Stammdatenmanagement sowie regionale Produktions-, Vertriebs- und Distributionseinheiten am Prozess beteiligt. Category Management, Warenbewirtschaftung und Stammdatenmanagement bilden die beteiligten Organsisationseinheiten auf Handelsseite ${ }^{2}$.

- Die Unternehmen tauschen die Produktstammdaten nicht einmalig, sondern beginnend in der Sortimentsplanungsphase in mehreren Schritten aus.

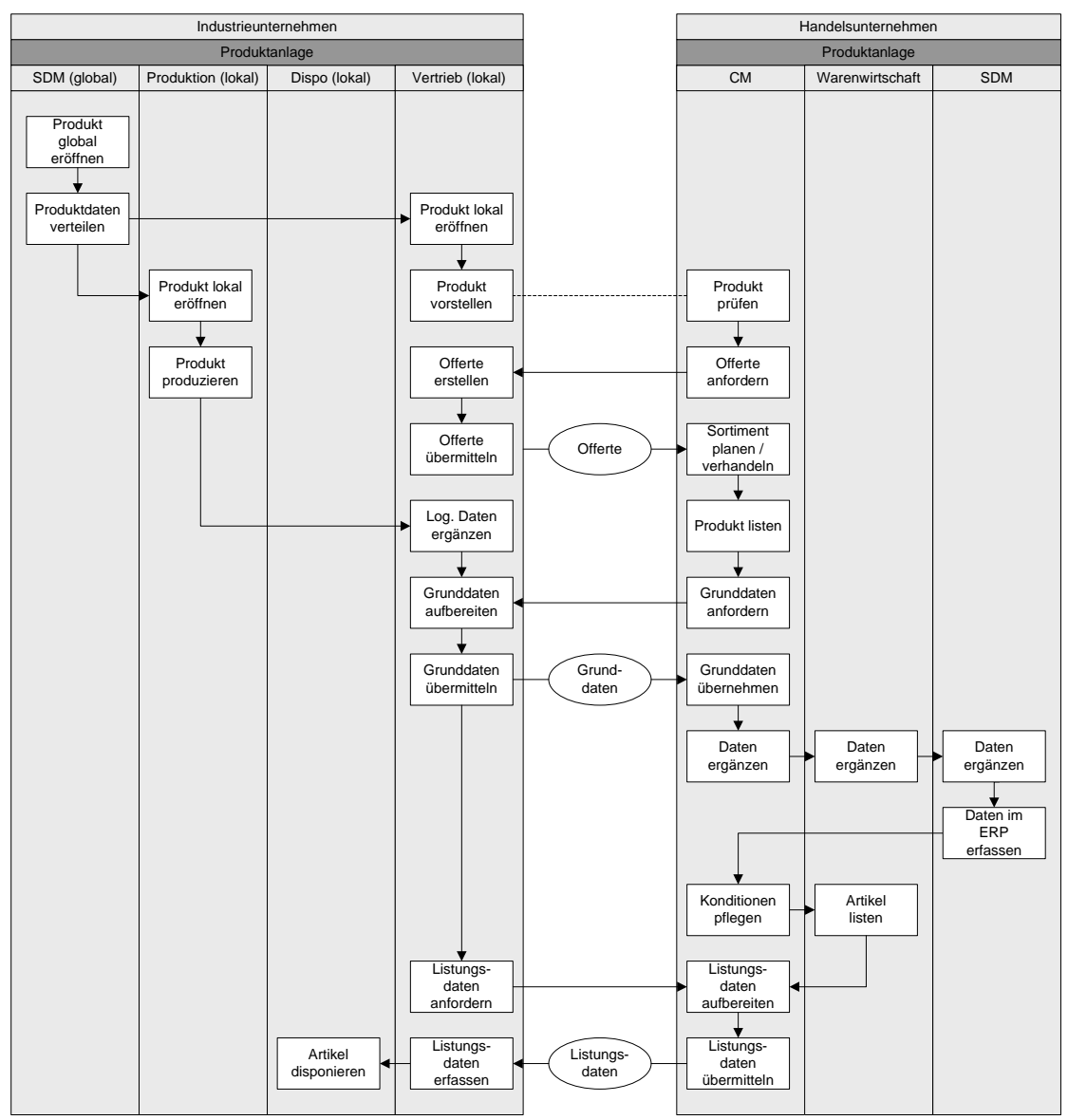

Legende: $\mathrm{SDM}=$ Stammdatenmanagement, Dispo $=$ Disposition, $\mathrm{CM}=$ Category Management

Abb. 3: Makro-Prozessablauf der Produktneuanlage von Markenprodukten

In regelmäßigen Zeitabständen, die vom Sortimentsplanungszyklus des Handels abhängen, treffen sich Vertriebsmitarbeiter des Herstellers und Category Manager

2 Das Handelsunternehmen ist zentral-dezentral organisiert (Becker u. Schütte 2004, Rotthowe 1998), d.h. die Einkaufs-, Warenwirtschafts- und Stammdatenfunktionen werden zentral ausgeführt. 
des Händlers, um die neue Produktpalette des Herstellers im Rahmen einer Produktvorstellung zu evaluieren. Vorgängig hat das Stammdatenmanagement auf Herstellerseite den globalen Produktstamm des Sortiments eröffnet, welchen der Vertrieb durch lokale Attribute ergänzte ${ }^{3}$.

Im Anschluss an die Produktvorstellung fordert das Category Management Offerten zu Teilen des vorgestellten Produktsortiments an. Der Vertrieb übermittelt dazu erste Produktstammdaten wie bspw. Produktnummern, Mengenverhältnisse logistischer Einheiten und Einkaufspreise an den Hersteller.

Basierend auf den vorliegenden Offerten plant das Category Management das Sortiment und fällt einen Listungsentscheid für bestimmte Produkte. Für die zu listenden Produkte fordert das Category Management eine Menge von ca. 30 Stammdatenattributen vom Hersteller an, insbesondere Artikelstruktur- und Logistikdaten.

Nach Übernahme der Herstellerdaten ergänzen die händlerseitigen Rollen die Stammdaten sukzessive um weitere ca. 50 händlerspezifische Attribute wie bspw. Listungs-, Verkaufs-, und POS-Daten, bevor das zentrale Stammdatenmanagement den Produktstamm im zentralen Warenwirtschaftssystem erfasst. Im Anschluss an die Konditionspflege durch das Category Management und die Artikellistung durch die Warenwirtschaft ist das Produkt im Warenwirtschaftssystem bestellbereit und das Category Management übermittelt bestimmte Listungsdaten wie bspw. Filialanzahl an den Hersteller, der diese primär für die Erstbestückung des VMI-Lagers benötigt.

\subsection{Systemarchitektur: Datenaustausch per Spreadsheet}

Abb. 4 veranschaulicht die Stammdatensysteme auf Seiten Hersteller und Handel und den systemseitigen Datenfluss beim überbetrieblichen Produktanlageprozess. Die Ist-Analyse macht deutlich, dass

- Industrie- und Handelsunternehmen intern zentrale Stammdatensysteme und eine Stammdatenverteilung in die dezentralen Systeme aufgebaut haben,

- sie Produktstammdaten unternehmensübergreifend vorwiegend in Form von manuell aufbereiteten Spreadsheet-Dateien austauschen.

3 Zur Unterscheidung von globalen vs. lokalen Prozessen und Stammdaten vgl. den folgenden Abschnitt 5.3. 


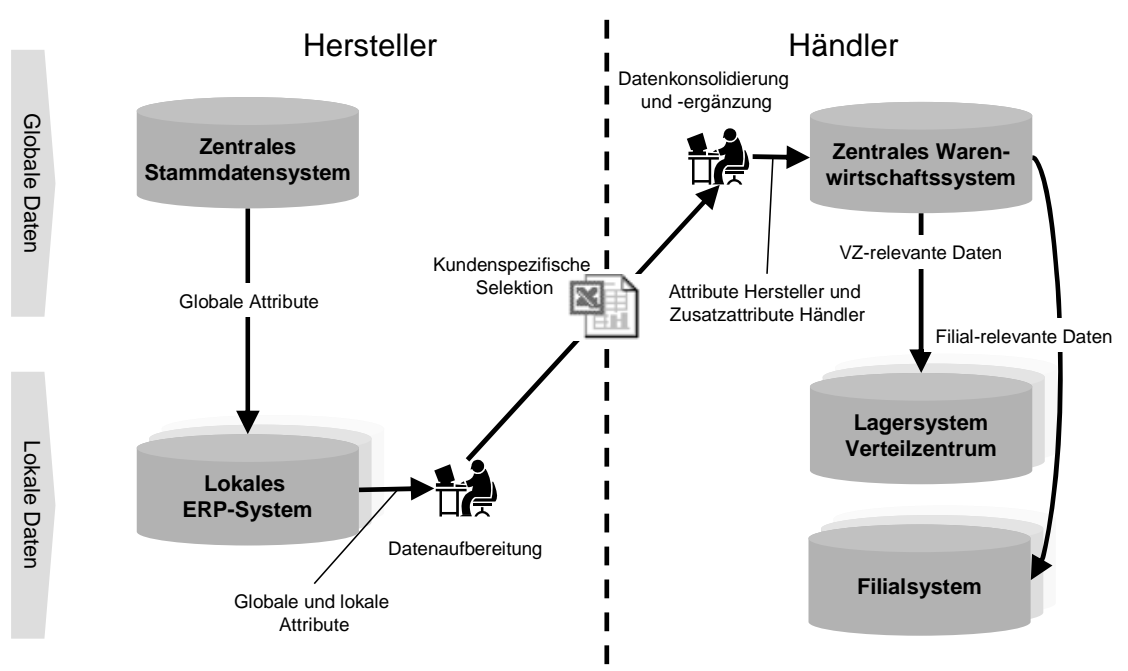

Abb. 4: Datenfluss der überbetrieblichen Produktanlage

Auf Seite der meist global agierenden Industrieunternehmen besteht eine klassische Aufteilung in globale und lokale Geschäftsprozesse und Daten (Marchand 2004, Pohland 2000): Sie konzentrieren bspw. die Produktentwicklung und den Einkauf typischerweise auf globaler Ebene, während Produktion und Vertrieb i.d.R. unabhängig innerhalb der lokalen Unternehmensbereiche ablaufen (Hess 1996, Pohland 2000). Die skizzierte Verteilung der Prozesse hat Einfluss auf die System- und damit auch die Stammdatenverteilung. Die Industrieunternehmen halten globale Produktstammdaten, d.h. Stammdatenattribute, die von globalen Prozessen oder von mehreren lokalen Prozessen analog verwendet werden, in einem zentralen Stammdatensystem. Es handelt sich hierbei i.d.R. um eine Instanz eines ERP-Systems, die lediglich für die Verwaltung der globalen Stammdatenattribute, wie bspw. Produktnummern, -beschreibungen, und -klassifikationen genutzt wird. Das zentrale Stammdatensystem verteilt die globalen Stammdaten regelmäßig in die ERP-Systeme der verschiedenen Produktions- und Vertriebsorganisationen. Die lokalen Organisationen ergänzen die globalen Stammdatenattribute um lokale Attribute wie bspw. logistische Informationen zur Steuerung ihrer lokalen Prozesse und Konditionen.

Auf Händlerseite bietet sich ein ähnliches Bild: Globale Stammdaten werden in einem zentralen Warenwirtschaftssystem gehalten, welches Teile der globalen Produktstämme an die Systeme in den Verteilzentren und Filialen verteilt. Im Gegensatz zur Industrie ist in dem untersuchten Handelsunternehmen der Anteil globaler Stammdatenattribute deutlich höher: die zentral-dezentrale Organisation und Prozessverteilung führt dazu, dass der Grossteil der Stammdatenattribute global geführt wird und nur wenige Attribute in den lokalen Systemen ergänzt werden. 
Die IT-Unterstützung des zwischenbetrieblichen Datenflusses beschränkt sich überwiegend auf Tabellenkalkulationsformate wie Microsoft Excel. Der direkte Stammdatenaustausch über standardisierte EDI- bzw. XML-Nachrichten oder über Stammdatenpools wird nur in einem sehr geringen Anteil der Partnerbeziehungen eingesetzt. Auf Herstellerseite übertragen Vertriebsmitarbeiter die händlerrelevanten Stammdatenattribute in spezielle Datenbanken, auf deren Basis sie die in den verschiedenen Prozessphasen vom Händler geforderten Produktstammdaten i.d.R. nach Händler-Vorgaben (Templates) kundenspezifisch aufbereiten. Das Category Management nimmt die per Email übermittelten Tabellen auf Händlerseite entgegen, konsolidiert die Daten verschiedener Hersteller und selektiert und ergänzt die Stammdaten je nach Bedarf vor der Erfassung im zentralen Warenwirtschaftssystem.

\section{Global Data Synchronization: Bewertung des Lösungsansatzes}

\subsection{Standardisierungsgrad und Anschlussfähigkeit als Bewertungsgrundlage}

Die Bewertung des GDSN-Ansatzes erfolgt anhand von zwei Dimensionen. Erstens wird die Reife von GDSN anhand des Standardisierungsgrads beurteilt: Um Produktstammdatenmanagement zwischen Industrie und Handel elektronisch zu unterstützen, müssen die beteiligten Unternehmen die gleiche Sprache sprechen, d.h. GDSN muss Daten, Funktionen, Prozesse und Geschäftsbeziehungen ausreichend standardisieren (Heutschi et al. 2004, Huber et al. 2002, Kagermann u. Österle 2006, McAfee 2005). Zweitens beurteilen wir anhand der vorgestellten Studie die Fähigkeit heutiger Unternehmen, die bestehenden GDSN-Standards zu bedienen. Zur Umsetzung des Konzepts müssen Hersteller und Händler ihre internen Anwendungen (Daten und Funktionen), Prozesse (Organisation) und Geschäftsmodelle so ausgestaltet haben, dass sie bezogen auf das Geschäftsnetzwerk anschlussfähig bzw. netzwerkfähig (Fleisch 2001) sind.

\subsection{Gestaltungsebene: Daten und Funktionen}

\subsubsection{Standardisierungsgrad}

Datenstandards vereinheitlichen die Struktur und die Bedeutung der übermittelten Daten (Heutschi et al. 2004). Die im GDSN verwendeten Datenstandards sind wie bspw. die GTIN bereits seit vielen Jahren im produktiven Einsatz. Obwohl bei Datenstandardisierungen immer Interpretationsspielräume bleiben, hat die Unter- 
suchung gezeigt, dass die GS1-Datenstandards nicht zuletzt aufgrund ergänzender Mappings auf die älteren EANCOM-Standards (GS1 2006a) und detaillierter Leitfäden für Spezialfragen wie Abmessungen (GS1 2006f) oder Produktnummernvergabe (GS1 2006d) größtenteils einheitlich interpretiert werden. Die Analyse des GDSN verdeutlicht hingegen ein anderes Problem, was in gewisser Weise typisch für Standardisierungsinitiativen ist. Im Fall der untersuchten Unternehmen fordert der Händler insgesamt ca. 30 Stammdatenattribute vom Hersteller an. Die GDSN-Standards sind extrem mächtig und definieren neben 11 Kernattributen weit über 200 weitere Ergänzungsattribute. Dadurch werden zwar die wichtigsten Anforderungen vieler Unternehmen abgedeckt, jedoch steigt der Aufwand für die Einarbeitung und bilaterale Abstimmung bei der konkreten Anwendung des Standards. Zukünftig sind auch im GDSN Initiativen zu erwarten, wie sie in der Schweiz bspw. in Form der EANCOM Ideal Messages (GS1 Schweiz 2006) praktiziert werden, d.h. die Reduzierung von Datenstandards auf einen sinnvollen, handhabbaren gemeinsamen Nenner.

Funktionsstandards liegen vor, wenn alle Stellen im Prozess die Daten nicht nur gleich verstehen, sondern zusätzlich die gleiche Verwendung sichergestellt ist (Kagermann u. Österle 2006, Reimers 2001), also z. B. eine Funktion „Stammdatenanlage“ die Durchführung von gewissen Validierungen oder Plausibilitätsprüfungen enthält. Das GDSN realisiert durch die Zentralisierung von Teilfunktionen in Datenpools und dem zentralen Verzeichnis einen gewissen Grad an funktionaler Standardisierung. So sind Funktionen wie bspw. die Registrierung von Geschäftspartnern oder Produkten im GDSN definiert und werden im Rahmen der Zertifizierung getestet. Die Spezifikation weiterer wichtiger Funktionen bspw. zur Validierung von Produktstammdaten lassen sich aktuell nicht finden.

\subsubsection{Anschlussfähigkeit}

Die Analyse zeigt, dass die Datenanforderungen der GDSN-Standards von den Industrie- und Handelsunternehmen in ihren Anwendungsarchitekturen zum Grossteil abbildbar sind.

Jedoch existieren spezifische Herausforderungen auf Seite des Handels bzw. der Hersteller. Beim Mapping auf das Datenmodell der verwendeten Anwendungen wurden einige Punkte, die bereits aus der Literatur bekannt sind (Schütte u. Vering 2004), bestätigt:

- Nicht sämtliche Warenwirtschaftssysteme unterstützen die Verwaltung von mehreren GTINs je Artikel bzw. logistischer Einheit

- Mit Ausnahme der Konditionen sind die Stammdatenattribute in Warenwirtschaftssystemen i.d.R. nicht mit einem Zeitbezug hinterlegt, so dass die Zeitsteuerung von Stammdatenänderungen organisatorisch verwaltet werden muss. 
- Die Anwendungen müssen parallele Produktklassifikationen in verschiedenen Produkthierarchien unterstützen, da nicht davon ausgegangen werden kann, dass Händler und auch Industrie ihre internen Klassifizierungssysteme zugunsten des GPC ablösen.

Für global operierende Industrieunternehmen ergeben sich Herausforderungen durch die bestehende Stammdatenverteilung (vgl. Abschnitt 5.3). Aufgrund lokaler Produktions- und Vertriebsprozesse liegt die Datenhoheit für große Anteile der Produktstammdaten i.d.R. in lokaler Verantwortung. Um einen globalen HomeDatenpool bedienen zu können, müssen die Unternehmen jedoch die lokalen Stammdatenattribute zumindest virtuell zentralisieren. Ein Lösungsansatz besteht im Einsatz dedizierter Produktstammdatenmanagementsysteme (Lucas-Nülle 2005, White 2005), die in Analogie zu den vorhandenen zentralen Stammdatensystemen die externe Verteilung von Produktstammdaten übernehmen. Neben der Zentralisierung sollten diese dedizierten Systeme zusätzliche Stammdatenmanagementfunktionen wie die Verwaltung partnerspezifischer Sichten auf Stammdaten, ein Monitoring der externen Datenverteilung und Workflow-Unterstützung der wesentlichen Stammdatenprozesse bieten.

Im Hinblick auf die Anschlussfähigkeit und die Akzeptanz des GDSN-Konzepts kommt der Benutzerfreundlichkeit der Stammdaten-bezogenen Anwendungen eine wesentliche Bedeutung zu. Die vom GDSN verfolgte elektronische Unterstützung des überbetrieblichen Stammdatenmanagements hat nur dann Aussicht auf Erfolg, wenn die Kopplung zwischen internen Stammdatensystemen und Datenpools so benutzerfreundlich und flexibel ist, dass sie von den beteiligten Vertriebsmitarbeitern, Einkäufern, etc. auch wirklich genutzt werden. Die starke Verbreitung von Tabellenkalkulationsprogrammen als Werkzeug in überbetrieblichen Stammdatenprozessen verdeutlicht den Bedarf an flexibler und einfacher Unterstützung.

Auf dem US-amerikanischen Markt bieten in jüngster Zeit Unternehmen wie bspw. Logical Apps, Optura, MDQ Systems oder BackOffice Associates spezialisierte sog. Packaged Composite Applications (Woods 2003) für das Stammdatenmanagement in Industrieunternehmen an. Die Lösungen bieten erweiterte Funktionen wie bspw. Datenmigration und -konsolidierung, Workflow-Unterstützung für Stammdatenprozesse und Datenqualitätsmanagement und -monitoring auf der Basis etablierter ERP-Systeme von SAP und Oracle (Swanton 2006). 


\subsection{Gestaltungsebene: Prozesse im Stammdatenmanagement}

\subsubsection{Standardisierungsgrad}

Prozessstandards stellen sicher, dass alle Geschäftspartner im Netzwerk einen bestimmten Geschäftsprozess gleich abwickeln (Heutschi et al. 2004, Kagermann u. Österle 2006). Analog zu vielen zwischenbetrieblichen Standards offenbart das GDSN in Bezug auf die Prozessstandardisierung eine „organisatorische Lücke“ (Huber et al. 2002, Kubicek 1992). Erst der Kontext der jeweiligen internen Stammdatenprozesse ermöglicht ein Verständnis des überbetrieblichen Prozesses und der entsprechenden Interaktion zwischen den Unternehmen. Zwar definiert das GDSN über den skizzierten Publish-Subscribe-Prozess die grundlegende Funktionsweise des Stammdatenaustauschs, die detaillierte Analyse des Produktanlageprozesses offenbarte jedoch zahlreiche offene Fragestellungen:

- Produktstammdaten werden nicht „en bloc“ übertragen, vielmehr werden sie in unterschiedlichen Umfängen im Rahmen der Sortimentsplanung sowie nach dem Listungsentscheid sukzessive zu unterschiedlichen Zeitpunkten übertragen und ergänzt. Die Analyse verdeutlichte, dass ein übergreifendes Verständnis über die zeitliche Abfolge von Datenanforderungen und -verwendung im Rahmen einer Produktanlage auf Industrie- und Handelsseite nur sehr eingeschränkt vorhanden war. So war der Industrieseite bspw. häufig nicht detailliert bekannt, zu welchen Zeitpunkten der Handel welchen Teil der Produktstammdaten in welcher Qualität bzw. Detaillierungsstufe benötigt.

- Neben der Produktanlage sind die weiteren Mikro-Prozesse im überbetrieblichen Stammdatenmanagement explizit zu definieren. Die Prozesse für Stammdatenänderungen und -ausphasierungen sind im Vergleich zur Anlage deutlich komplexer und überbetrieblich äußerst unzureichend definiert.

- Die Stammdatenprozesse variieren zwischen Markenartikeln und Eigenmarken oder aufgrund spezifischer Eigenschaften der Sortimente. Ein überbetrieblicher Prozessentwurf müsste die Prozesse soweit wie möglich harmonisieren und die verbleibenden notwendigen Varianten spezifizieren.

\subsubsection{Anschlussfähigkeit}

Die elektronische Abwicklung eines überbetrieblichen Prozesses erfordert i.d.R. interne Umstellungen. Die Analyse des überbetrieblichen Produktanlageprozesses verdeutlicht, dass interne Stammdatenprozesse in Industrie und Handelsunternehmen bspw. im Vergleich zu Logistikprozessen deutlich schwächer strukturiert und automatisiert sind. Die Stammdatenanlage variiert unternehmensintern abhängig von Sortimenten sowie Geschäftsbereichen und die Koordination verschiedener Rollen geschieht „auf Zuruf“. Die Definition der Stammdatenprozesse, insbeson- 
dere für Änderungen und Ausphasierung, und deren Unterstützung durch einen elektronischen Workflow ist in vielen Unternehmen erst im Anfangsstadium. Erst wenn die internen Prozesse auf Industrie- und Handelsseite definiert und die Synchronisationspunkte zum überbetrieblichen Prozess klar sind, hat die angestrebte überbetriebliche Automatisierung im GDSN Aussicht auf Erfolg und läuft nicht intern hinter den Unternehmensgrenzen ins Leere.

\subsection{Gestaltungsebene: Geschäftsstandards}

\subsubsection{Standardisierungsgrad}

Geschäftsstandards regeln die Zusammenarbeit der an einem kooperativen Prozess beteiligten Unternehmen, bspw. durch Service-Level-Agreements oder die Klärung von Haftungsfragen (Kagermann u. Österle 2006). Die „organisatorische Lücke“ der Prozessebene im GDSN zieht sich in der Geschäftsebene durch. Die Durchsetzung des Standards hängt entscheidend von der Klärung von Verantwortlichkeiten bspw. für Plausibilitätsprüfungen ab. Haftungsvereinbarungen für Fehlerfälle, umfassende Service Level Agreements und allgemein akzeptierte Definitionen von Führungsgrößen für das kooperative Stammdatenmanagement sind zentrale Voraussetzungen.

\subsubsection{Anschlussfähigkeit}

Industrie- und Handelsunternehmen werden sich nur an das GDSN anschließen, wenn sie einen klaren betriebswirtschaftlichen Mehrwert erkennen. Die Realisierung der Anschlussfähigkeit an GDSN auf Daten-, Funktions- und Prozessebene erfordert erhebliche informationstechnische und organisatorische Investitionen. Demgegenüber stehen Nutzenpotenziale einerseits aufgrund der Verfügbarkeit und Qualität der Stammdaten, die über GDSN zur Verfügung gestellt werden, andererseits aus der Reduktion der Kosten sowie des Aufwands für die Übertragung von Stammdaten zwischen Industrie und Handel. Zwar existieren dazu jeweils Abschätzungen, jedoch fehlt der Nachweis der Auswirkungen auf grundlegende Messgrößen wie Logistikkosten und Regalverfügbarkeit.

Zusätzlich bleibt abzuwarten, wie die Mehrheit der Unternehmen die mit dem GDSN verbundene Zentralisierung von Stammdaten in Pools bewertet. Die Nutzung von Datenpools ist mit zusätzlichen Kosten verbunden, wird den bilateralen Austausch z. B. im Falle von Preisen und Konditionen aber nie ganz ersetzen. Zusätzlich zeigen Erfahrungen in anderen Branchen, dass der Besitz von Stammdaten eine gewisse Machtposition in Geschäftsnetzwerken bedeutet (Kagermann u. Österle 2006). Es bleibt abzuwarten, ob Industrie- und Handelsunternehmen bereit sind, Teile dieser Wettbewerbsstärke an Dritte (wenn auch konsortial geführt) abzugeben. Schließlich hängt der Erfolg von GDSN aufgrund von Netz- 
werkeffekten von einem schnellen Wachstum der Anzahl angeschlossener Unternehmen ab (Shapiro u. Varian 1998).

\section{Zusammenfassung und Ausblick}

GDSN wird aktuell als Lösungsansatz zur Umsetzung eines m:n-fähigen elektronischen Stammdatenmanagements zwischen Industrie und Handel propagiert. Die Ist-Analyse bei zwei europäischen Industrie- und einem Handelsunternehmen zeigt, dass überbetriebliche Stammdatenprozesse derzeit wenig abgestimmt sind, mehrstufige Interaktionen umfassen und verschiedene Rollen innerhalb der Unternehmen involvieren. Wie sich aus anderen Szenarien der elektronischen Kooperation übertragen lässt, erfordert die elektronische Abwicklung eine überbetriebliche Abstimmung auf Ebene der Geschäftsstandards, der Prozesse sowie der Daten und Funktionen. Für die Durchsetzung des GDSN-Konzepts ist daher einerseits die Reife der aktuellen Standardisierung in Bezug auf diese Ebenen, andererseits die Anschlussfähigkeit der Unternehmen an diese Standardisierung zu bewerten. Tab. 3 fasst die Bewertung des GDSN-Konzepts zusammen.

\begin{tabular}{|l|l|l|}
\hline Gestaltungsebene & $\begin{array}{c}\text { Reife von GDSN } \\
\text { (Standardisierungsgrad) }\end{array}$ & $\begin{array}{c}\text { Anschlussfähigkeit } \\
\text { der Unternehmen }\end{array}$ \\
\hline Geschäftsstandard & unzureichend & unzureichend \\
\hline Prozess & ausbaufähig & unzureichend \\
\hline Funktion & ausbaufähig & ausbaufähig \\
\hline Daten & ausreichend & ausbaufähig \\
\hline
\end{tabular}

Tab. 3: Überblick der Bewertung des GDSN

GDSN verfügt über mächtige Datenstandards, die aufgrund ihrer Historie in Industrie und Handel weit verbreitet und akzeptiert sind. Reale Produktstammdatenprozesse und die unterstützenden Funktionen sind vielfältiger und lassen sich nicht ausschließlich auf das im Standard definierte Publish-Subscribe-Pattern zurückführen. Folglich bilden organisatorische Ergänzungen des Konzepts, d.h. die Definition der überbetrieblichen Prozessinteraktion sowie Geschäftsstandards eine Voraussetzung für erfolgreiche Implementierungen.

Die untersuchten Unternehmen sind heute noch nicht in der Lage, das GDSNKonzept umzusetzen. Dies erfordert Anpassungen der internen Daten- und Anwendungsarchitekturen sowie elektronische Workflows für Produktanlage, -änderung und -ausphasierung, die sich über den Home-Datenpool mit den überbetrieblichen Prozessen koppeln lassen. Diese unter Umständen langwierigen internen Aufräumarbeiten sind Voraussetzung für ein überbetriebliches, elektronisches Produktstammdatenmanagement. Sie könnten sich jedoch durch Nutzung der aufkommenden dedizierten Systeme zum Master Data Management oder Product Information Management reduzieren. 
Sofern die genannten Punkte adressiert werden, bietet sich GDSN als Konzept der Zukunft an. Ob es sich als zukünftige Lösung durchsetzen wird, hängt allerdings nicht zuletzt von strategischen Überlegungen der Marktteilnehmer, deren Anschlussfähigkeit und der damit verbundenen Diffusionsgeschwindigkeit des Standards ab. 


\section{Literaturverzeichnis}

Alt R, Fleisch E (1999) Key Success Factors in Designing and Implementing Business Networking Systems. In: Klein, S, Gricar, J, Pucihar, A (Hrsg): 12th Bled Electronic Commerce Conference: Global Networked Organizations, Bd 1: Research. Moderna organizacija, S 219-235

Becker J, Schütte R (2004) Handelsinformationssysteme. Redline Wirtschaft, Frankfurt am Main

Bowling T, Licul E, Van Hammond D (2004) Global Data Synchronization: Building a flexible approach. IBM Institute for Business Value, Somers, S

Corsten DS, Gruen T (2003) Desperately seeking shelf-availability: an examination of the extent, the causes, and the efforts to address retail out-of-stocks. International Journal of Retail \& Distribution Management, Bd 31, Ausg 12, S 605-617

Corsten DS, Hofstetter JS (2001) After the hype: The emerging landscape of B2B exchanges. ECR Journal, Bd 1, Ausg 1, S 51-59

Fleisch E (2001) Das Netzwerkunternehmen. Strategien und Prozesse zur Steigerung der Wettbewerbsfähigkeit in der "Networked Economy". Springer, Berlin et al.

Garf R, Romanow K (2005) GNX and WWRE Finalize Merger: Say Hello to Agentrics. AMR Research, Boston

Global Commerce Initiative, Capgemini (2005) Global Data Synchronisation At Work in the Real World - Illustrating the Business Benefits. Paris

Grocery Manufacturers of America, Food Marketing Institute, A. T. Kearney (2003) Action Plan to Accelerate Trading Partner Electronic Collaboration.

GS1 (2004) GDSN Introductory Brochure. Brüssel

GS1 (2006a) Catalogue Item Synchronization BMS 9.0.3 to EANCOM 2002 Mapping (Draft Document Version 0.2). Brüssel

GS1 (2006b) GDSN Statistics of Adoption. http://www.gs1.org/docs/gdsn/gdsn_adoption.pdf, Abrufdatum: 31.03.2006

GS1 (2006c) GS1 XML. Abrufdatum: 31.03.2006

GS1 (2006d) GTIN Allocation Rules. http://www.gs1.org/gtinrules, Abrufdatum: 31.03.2006

GS1 (2006e) List of GDSN certified Data Pools. http://www.gs1.org/docs/gdsn/gdsn_certified_data_pools.pdf, Abrufdatum: 31.03.2006 
GS1 (2006f) Package Measurment Rules for Data Alignment. Brüssel

GS1 Schweiz (2006) EANCOM Ideal Messages. http://www.gs1.ch/produktedienstleistungen/ProdukteDienstleistungen/GS1Syste m/eCommerceEDI/EANCOMIdealMessages/tabid/752/Default.aspx, Abrufdatum: 31.03.2006

Hertel J, Zentes J, Schramm-Klein H (2005) Supply-Chain-Management und Warenwirtschaftssysteme im Handel. Springer, Berlin

Hess T (1996) Entwurf betrieblicher Prozesse - Grundlagen, Bestehende Methoden, Neue Ansätze. Gabler, Wiesbaden

Heutschi R, Leser F, Erni F, Alt R, Österle H (2004) WebService-Technologien als Enabler des Real-time Business. In: Alt, R, Österle, H (Hrsg): Real-time Business: Lösungen, Bausteine und Potentiale des Business Networking. Springer, Berlin, S 133-155

Holzkämper O (1999) Category Management: Strategische Positionierung des Handels. Universität Göttingen, Göttingen

Huber T, Alt R, Lehmann G (2002) Templates: Standardisierung beim Business Networking. In: Österle, H, Fleisch, E, Alt, R (Hrsg): Business Networking in der Praxis. Springer, Berling, S 251-269

Kagermann H, Österle H (2006) Geschäftsmodelle 2010 - Wie CEOs Unternehmen transformieren. Frankfurter Allgemeine Buch, Frankfurt

Kubicek H (1992) The Organization Gap in Large-Scale EDI Systems. In: Streng, RJ, Ekering, CF, van Heck, E, Schultz, JFH (Hrsg): Scientific Research on EDI "Bringing Worlds Together". Samsom, Amsterdam, S 11-41

Leukel J (2004) Katalogdatenmanagement im B2B E-Commerce. Josef Eul Verlag, Lohmar

Loderhose B (2005a) Ahold forciert synchrone Daten. Lebensmittelzeitung, Ausg 16, S 26

Loderhose B (2005b) Briten synchronisieren Stammdaten. Lebensmittelzeitung, Ausg 14, S 26

Loos P (1999) Grunddatenverwaltung und Betriebsdatenerfassung als Basis der Produktionsplanung und -steuerung. In: Corsten, H, Friedl, B (Hrsg): Produktionscontrolling. Vahlen, München

Lucas-Nülle T (2005) Product Information Management in Deutschland. plV pro literatur Verlag Robert Mayer-Scholz, Mammendorf

Marchand DA (2004) Seeking Global Advantage with Information Management and Information Technology Capabilities. In: Lane, HW, Maznevski, ML, Mendenhall, ME, McNett, J (Hrsg): The Blackwell Handbook of Global Management. Blackwell Publishing, Oxford, S 300-321 
McAfee A (2005) Will Web Services Really Transform Collaboration? MIT Sloan Management Review, Bd 46, Ausg 2, S 78-84

Mertens P (1995) Integrierte Informationsverarbeitung 1 - Administrations- und Dispositionssysteme in der Industrie. Gabler, Wiesbaden

Nakatani K, Chuang T-T, Zhou D (2006) Data Synchronization Technology: Standards, Business Values and Implications. Communications of AIS, Ausg 17, S 2-60

Österle H (1995) Business Engineering: Prozess- und Systementwicklung, Band 1: Entwurfstechniken. Springer, Berlin

Pohland S (2000) Globale Unternehmensarchitekturen: Methode zur Verteilung von Informationssystemen. Weissensee Verlag, Berlin

Reimers K (2001) Standardizing the new e-business platform: Learning from the EDI experience. Electronic Markets, Bd 11, Ausg 4, S 231-237

Rode J (2005a) Nestlé will die Wege verkürzen. Lebensmittelzeitung, Ausg 39, S 26

Rode J (2005b) Pools im globalen Netz. Lebensmittelzeitung, Ausg 35, S 78

Rode J (2005c) Wal-Mart will Daten über globales Netz. Lebensmittelzeitung, Ausg 22, S 30

Rotthowe T (1998) Schnittstellen-Management im Handel: eine Analyse der Informationsflüsse zwischen Warenwirtschaft und Rechnungswesen. Gabler, Wiesbaden

Schütte R, Vering O (2004) Erfolgreiche Geschäftsprozesse durch standardisierte Warenwirtschaftssysteme - Marktanalyse, Produktübersicht, Auswahlprozess. Springer, Berlin

Senger E (2004) Zum Stand der elektronischen Kooperation - Fallstudien, Muster und Handlungsoptionen. Universität St. Gallen, St. Gallen

Shapiro C, Varian HR (1998) Information Rules - A Strategic Guide to the Network Economy. Harvard Business School Press, Boston/Massachusetts

Sparks L, Wagner BA (2003) Retail exchanges: a research agenda. Supply Chain Management, Bd 8, Ausg 3, S 201-108

Swanton B (2006) MDM on a Single ERP Instance: Workflow and Data Quality. AMR Research, Boston

Thonemann U, Behrenbeck K, Küpper J, Magnus K-H (2005) Supply Chain Excellence im Handel - Trends, Erfolgsfaktoren und Best-Practice-Beispiele. Gabler, Wiesbaden

Wedekind H (1997) Bestandsdaten, Bewegungsdaten, Stammdaten. In: Mertens, P, Back, A, Becker, J, König, W, Krallmann, H, Rieger, B, Scheer, A-W, Seibt, D, Stahlknecht, P, Strunz, H, Thome, R, Wedekind, H (Hrsg): Lexikon der Wirtschaftsinformatik. Springer, Berlin, S 61 
White A (2005) Magic Quadrant for Product Information Management. Gartner Group, Stamford

White A, Newman D, Logan D, Radcliffe J (2006) Mastering Master Data Management. Gartner Group, Stamford

White A, Prior D, Radcliffe J, Wood B, Holincheck J (2004) Emergence of EIM Drives Semantic Reconciliation. Gartner Group, Stamford

Woods D (2003) Packaged Composite Applications. O'Reilly, London 


\section{Autorenverzeichnis}

\section{Dr. Christine Legner}

Universität St. Gallen

Institut für Wirtschaftsinformatik

Müller-Friedberg-Str. 8, CH-9000 St. Gallen

Tel: +41 (0)71/224 2782, Fax: +41 (0)71/224 2777

E-Mail: christine.legner@unisg.ch

http://web.iwi.unisg.ch/org/iwi/iwi_web.nsf/wwwTeamGer/

LegnerChristine.htm

\section{Prof. Dr. Hubert Österle}

Universität St. Gallen

Institut für Wirtschaftsinformatik

Müller-Friedberg-Str. 8, CH-9000 St. Gallen

Tel: +41 (0)71/224 2420, Fax: +41 (0)71/224 2777

E-Mail: hubert.oesterle@unisg.ch

http://web.iwi.unisg.ch/org/iwi/iwi_web.nsf/wwwTeamGer/

OesterleHubert.htm

\section{Jan W. Schemm}

Universität St. Gallen

Institut für Wirtschaftsinformatik

Müller-Friedberg-Str. 8, CH-9000 St. Gallen

Tel: +41 (0)71/224 2787, Fax: +41 (0)71/224 2777

E-Mail: jan.schemm@unisg.ch

http://web.iwi.unisg.ch/org/iwi/iwi_web.nsf/wwwTeamGer/SchemmJan.htm 